\title{
European Option Pricing with Transaction Costs in Lévy Jump Environment
}

\author{
Jiayin Li, ${ }^{1}$ Huisheng Shu, ${ }^{1}$ and Xiu Kan ${ }^{2}$ \\ ${ }^{1}$ School of Science, Donghua University, Shanghai 200051, China \\ ${ }^{2}$ College of Electronic and Electrical Engineering, Shanghai University of Engineering Science, Shanghai 201620, China \\ Correspondence should be addressed to Huisheng Shu; hsshu@dhu.edu.cn
}

Received 25 January 2014; Accepted 20 February 2014; Published 27 March 2014

Academic Editor: Bo Shen

Copyright (c) 2014 Jiayin Li et al. This is an open access article distributed under the Creative Commons Attribution License, which permits unrestricted use, distribution, and reproduction in any medium, provided the original work is properly cited.

\begin{abstract}
The European option pricing problem with transaction costs is investigated for a risky asset price model with Lévy jump. By the aid of arbitrage pricing theory and the generalized Itô formula (which includes Poisson jump), the explicit solution to the risk asset price model is given. According to arbitrage-free principle, we first discretize the continuous-time model. Then, in each small time interval, the transaction costs are introduced. By using the $\Delta$-hedging strategy, the explicit solutions of the European options pricing formula with transaction costs are given for the risky asset price model with Lévy jump.
\end{abstract}

\section{Introduction}

Recently, the stochastic differential equation theory has found more and more applications in many fields such as finance [1$12]$ and control and filtering [13-25]. The option pricing with transaction costs has been one of the important problems and received increasing research attention. The development of the option pricing problem is reviewed as follows. In [1], the hedge strategy has been introduced initially to solve the option pricing problem with transaction costs. In [5], the option pricing problem with transaction costs has been transformed into a stochastic optimal control issue. Subsequently, a perfect hedge strategy has been proposed in [8] to deal with CRR model with transaction costs and a discrete algorithm has been given. In [26], the transaction costs of European options have been proposed on the discrete time points for three scenarios, that is, without transaction costs, with proportional transaction costs and with concave transaction costs. By using the stochastic dominance theory, in [7], the upper/lower limit has been given for European call option with transaction costs as well as its corresponding volatility. In order to avoid solving a complicated stochastic optimization problem, in [27], an effective algorithm on
Markov chain has been proposed to obtain the European option price. For the case of random volatility, a nonlinear differential equation has been constructed in [28] to price the European option with transaction costs. Some efforts have also been made on the American option pricing problems. For example, the American option pricing problem has been studied for the jump diffusion model with transaction costs in [29], where the problem addressed has first been transformed into a binary stochastic control problem and then solved numerically.

It should be pointed out that, in the frictional financial market, the models mentioned above have not taken the risks into account. For the pricing problem with transaction costs, we also need to consider the risk asset pricing model. Since the B-S [2] option pricing formula put forward, option pricing has been an important part of financial mathematics. As early as 1976, Merton [11] noticed that when some major message occurred, the risky asset price's changing was discontinuous and pointed out that the risky asset was driven by a Brownian motion and a jump diffusion model. Aase [30] presented Itô process and random point process mixture model. Scott [4] built a jump-diffusion model with stochastic volatility and interest rate and gave the European option 
pricing formula. Chan's [31] came up with Lévy process model and obtained the option pricing formula.

In this paper, the risk asset pricing model with Lévy jump diffusion is considered and, following Leland's [1] idea, a more realistic pricing formula is given that has more practical potential.

\section{Problem Formulation and Preliminaries}

The B-S model has been proposed in [2] where the B-S model based European option pricing formula has been obtained. In [11], the Poisson jump diffusion process has been introduced to represent the stock price process, and the corresponding European option pricing formula has been derived. In this paper, we will investigate the European option pricing problem for the Merton's model.

The following assumption is needed.

Assumption 1. Suppose that the following conditions are satisfied:

(i) risk-free rate $r$ is a constant;

(ii) there is no dividend;

(iii) there are no transaction costs;

(iv) there is no arbitrage opportunity.

Consider the following asset model [32]:

$$
\frac{d S(t)}{S(t-)}=\mu d t+\sigma d W(t)+\int_{-1}^{\infty} y N(d t, d y)
$$

where $S(t)$ is the price of risky asset; suppose $S(t)$ has a jump at time $t$, and then $y$ is the size of this jump, which is also $y=S(t)-S(t-) ; \mu$ represents the rate of return; $\sigma$ denotes the volatility; $W(t)$ is a standard Brownian motion; $N(d t, d y)$ is a Poisson measure with its intensity $v(d t, d y)$.

From (1), we further have

$$
S(t)=S(0) \exp \left\{\sigma W(t)+\left(r-\frac{1}{2} \sigma^{2}\right) t\right\} \prod_{i=1}^{N(t)}\left(U_{i}+1\right)
$$

where $r$ is risk-free rate.

We introduce the following lemmas that will be used to obtain the main results.

Lemma 2 (see [33]). Let $Y(t) \in \mathbb{R}$ be a Lévy-Itô integration described as follows:

$$
\begin{aligned}
d Y(t)= & G(t) d t+F(t) d W(t)+\int_{|x|<1} H(t, x) \widetilde{N}(d t, d x) \\
& +\int_{|x| \geq 1} K(t, x) N(d t, d x)
\end{aligned}
$$

where $\widetilde{N}(d t, d x)$ is a compensated Poisson measure and the continuous part $Y^{c}(t)$ of process satisfies

$$
d Y^{c}(t)=G(t) d t+F(t) d W(t) .
$$

Then, for any $f \in C^{2}(\mathbb{R} \times \mathbb{R}), t \geq 0, f(Y(t))$ is also a Lévy-Itô integration and satisfies

$$
\begin{aligned}
& f(Y(t))-f(Y(0)) \\
& =\int_{0}^{t} \frac{\partial f}{\partial y}(Y(s-)) d Y^{c}(s) \\
& \quad+\frac{1}{2} \int_{0}^{t} \frac{\partial^{2} f}{\partial y^{2}}(Y(s-)) d\left[Y^{c}, Y^{c}\right](s) \\
& +\int_{0}^{t} \int_{|x| \geq 1}[f(Y(s-)+K(s, x))-f(Y(s-))] N(d s, d x) \\
& \quad+\int_{0}^{t} \int_{|x| \geq 1}[f(Y(s-)+H(s, x))-f(Y(s-))] \widetilde{N}(d s, d x) \\
& \quad+\int_{0}^{t} \int_{|x|<1}[f(Y(s-)+H(s, x))-f(Y(s-)) \\
& \left.-H(s, x) \frac{\partial f}{\partial y}(Y(s-))\right] v(d x) d s .
\end{aligned}
$$

Set

$$
\int_{0}^{\infty} \int_{-1}^{\infty} y N(d t, d y)=\sum_{j=1}^{N(t)} U_{j}
$$

with probability, and denote

$$
N(t)=\sum_{m=1}^{M} N_{m}(t)
$$

where $N(t), N_{1}(t), \ldots, N_{M}(t), 0 \leq t \leq T$ are Poisson processes with respective intensities $\lambda, \lambda_{1}, \ldots, \lambda_{M}$ and $\left(U_{j}\right)_{j \geq 1}$ represents the independent and identically distributed random variables taking values in the set $\left\{y_{1}, \ldots, y_{M}\right\}$.

Defining $p\left(y_{m}\right)=\lambda_{m} / \lambda$, we have the following lemma.

Lemma 3 (see [34]). Let $V(t, S)$ be the risk-neutral price of a European call paying $V(T, S(T))=(S(T)-K)^{+}=\max (S(T)-$ $K, 0)$ at time $T$, where $K$ is a strike price. If the risky asset price model satisfies (1), then we have

$$
\begin{aligned}
\frac{\partial V}{\partial t}+ & r S \frac{\partial V}{\partial S}+\frac{1}{2} \sigma^{2} S^{2} \frac{\partial^{2} V}{\partial S^{2}} \\
& +\lambda\left[\sum_{m=1}^{M} p\left(y_{m}\right) V\left(t,\left(y_{m}+1\right) S\right)-V(t, S)\right]-r V=0
\end{aligned}
$$

Lemma 4. One has $\forall 0 \leq t<T$, the call option's final value condition is $V(T, S(T))=(S(T)-K)^{+}=\max (S(T)-K, 0)$. Then, with the help of Lemma 2.3.4 in [34], one has

$$
V(t, S(t))=c(t, S(t)),
$$


where $c(t, S(t))$ is the call option price given by

$$
\begin{aligned}
c(t, S(t))=\sum_{j=0}^{\infty} e^{-\lambda(T-t)} \frac{\lambda^{j}(T-t)^{j}}{j !} \mathbb{E}[ & x N\left(d_{+}(\tau, x)\right) \\
& \left.-K e^{-r \tau} N\left(d_{-}(\tau, x)\right)\right],
\end{aligned}
$$

with $d_{ \pm}=\left(\log (S / K)+\left(r \pm(1 / 2) \sigma^{2}\right)(T-t)\right) / \sigma \sqrt{T-t}$.

In an unstable financial market, investors use some financial instruments to hedge risks and price its value. The basic idea is to construct a portfolio to hedge risks. Similarly, $\Delta$-hedge can also be used in the process of pricing option. We take the risky asset price model following the geometric Brownian motion, for example, and describe the $\Delta$-hedge idea about the derivation of option pricing formula.

Assume that the asset price model is given by

$$
\frac{d S(t)}{S(t)}=\mu d t+\sigma d W(t) \text {. }
$$

Suppose that there is no arbitrage opportunity. We construct a portfolio $\Pi:=V-\Delta S$, where $\Delta$ is the shares of risky asset. Note that, in the interval $(t, t+d t), \Pi$ is not risky and the $\Delta$ is not changeable. Hence, the return rate of portfolio can be given by

$$
\frac{\Pi_{t+d t}-\Pi_{t}}{\Pi_{t}}=r d t
$$

Then, we have

$$
d V(t)-\Delta d S(t)=r \Pi_{t} d t=r(V-\Delta S) d t .
$$

On the other hand, by using Itô formula, it can be obtained that

$$
d V(t)=\left(\frac{\partial V}{\partial t}+\frac{1}{2} \sigma^{2} S^{2} \frac{\partial^{2} V}{\partial S^{2}}+\mu S \frac{\partial V}{\partial S}\right) d t+\sigma S \frac{\partial V}{\partial S} d W(t)
$$

Substituting (14) into (13) yields

$$
\begin{aligned}
\left(\frac{\partial V}{\partial t}\right. & \left.+\frac{1}{2} \sigma^{2} S^{2} \frac{\partial^{2} V}{\partial S^{2}}+\mu S \frac{\partial V}{\partial S}-\Delta \mu S\right) d t \\
& +\left(\sigma S \frac{\partial V}{\partial S}-\Delta \sigma S\right) d W(t)=r(V-\Delta S) d t .
\end{aligned}
$$

Since there is no risk, the coefficient of the random term $d W(t)$ in (15) should be zero; that is,

$$
\Delta=\frac{\partial V}{\partial S}
$$

and hence (15) becomes

$$
\frac{\partial V}{\partial t}+\frac{1}{2} \sigma^{2} S^{2} \frac{\partial^{2} V}{\partial S^{2}}+r S \frac{\partial V}{\partial S}-r V=0 .
$$

Finally, according to the condition of the final value $V(T, S(T))=(S(T)-K)^{+}=\max (S(T)-K, 0)$, we can obtain the call option pricing formula

$$
c(t, S)=S N\left(d_{+}\right)-K e^{-r(T-t)} N\left(d_{-}\right),
$$

where $d_{ \pm}=\left(\log (S / K)+\left(r \pm(1 / 2) \sigma^{2}\right)(T-t)\right) / \sigma \sqrt{T-t}$.

\section{Main Results}

As an earlier derivation of option pricing is implemented under some "idealised" conditions, its applications are limited. In order to loosen these restrictions, the transaction costs are introduced in the derivation of option pricing. For example, the hedging strategy has been considered in [2] when transaction costs occur at discrete time points, and the explicit formula of the call option price with transaction costs has been put forward. In [29], the European option pricing problem with the transaction costs has been studied for the jump diffusion model. In this section, we aim to give the European option pricing formula with transaction costs for the Lévy jump case.

In the interval $(t, t+\delta t)$ with a sufficiently small $\delta>0$, the profit is given by

$$
\delta \Pi=\delta V-\Delta \delta S-k|v| S
$$

where $k$ is the transaction cost ratio and $v$ is the number of risky asset changing. Hence, it is easily known that the transaction costs are $k|v| S$.

Our main results are given as follows.

Theorem 5. For the risky asset price model $(1), V(t, S)$ satisfies

$$
\begin{aligned}
\frac{\partial V}{\partial t}+ & r S \frac{\partial V}{\partial S}+\frac{1}{2} \sigma^{2} S^{2} \frac{\partial^{2} V}{\partial S^{2}}-k \sigma S^{2}\left|\frac{\partial^{2} V}{\partial S^{2}}\right| \sqrt{\frac{2}{\pi \delta t}} \\
& -k S\left|\frac{\partial^{2} V}{\partial S^{2}}\right| \frac{\mathbb{E}\left|\int_{-1}^{\infty} y S(t-) N(\delta t, \delta y)\right|}{\delta t} \\
& +\lambda\left[\sum_{m=1}^{M} p\left(y_{m}\right) V\left(t,\left(y_{m}+1\right) S\right)-V(t, S)\right]-r V=0 .
\end{aligned}
$$

Proof. By using Taylor's formula, it follows from (16) that

$$
\begin{aligned}
v= & \Delta(S+\delta S, t+\delta t)-\Delta(S, t) \\
= & \frac{\partial V}{\partial S}(S+\delta S, t+\delta t)-\frac{\partial V}{\partial S}(S, t) \\
= & \frac{\partial V}{\partial S}(S, t)+\delta S \frac{\partial^{2} V}{\partial S^{2}}(S, t)+\delta t \frac{\partial^{2} V}{\partial S \partial t}(S, t) \\
& \quad+o(\delta t)-\frac{\partial V}{\partial S}(S, t) \\
= & \delta S \frac{\partial^{2} V}{\partial S^{2}}(S, t)+\delta t \frac{\partial^{2} V}{\partial S \partial t}(S, t)+o(\delta t),
\end{aligned}
$$




$$
\begin{aligned}
v= & \frac{\partial^{2} V}{\partial S^{2}}(\mu S \delta t+\sigma S \delta W(t) \\
& \left.+\int_{-1}^{\infty} y S(t-) N(\delta t, \delta y)\right) \\
& +\delta t \frac{\partial^{2} V}{\partial S \partial t}(S, t)+o(\delta t)
\end{aligned}
$$

Then, we have

$$
v \approx \sigma S \frac{\partial^{2} V}{\partial S^{2}} \delta W(t)+\frac{\partial^{2} V}{\partial S^{2}} \int_{-1}^{\infty} y S(t-) N(\delta t, \delta y)
$$

According to no arbitrage principle $\mathbb{E}(\delta \Pi)=r \Pi \delta t$ and Lemma 2, we have

$$
\begin{aligned}
& \mathbb{E}(\delta \Pi)=\left(\frac{\partial V}{\partial t}+\frac{1}{2} \sigma^{2} S^{2} \frac{\partial^{2} V}{\partial S^{2}}\right. \\
&\left.+\lambda\left[\sum_{m=1}^{M} p\left(y_{m}\right) V\left(t,\left(y_{m}+1\right) S\right)-V(t, S)\right]\right) \delta t \\
&-k \sigma S^{2} \mathbb{E}\left|\frac{\partial^{2} V}{\partial S^{2}} \delta W(t)\right| \\
&-k S \mathbb{E}\left|\frac{\partial^{2} V}{\partial S^{2}} \int_{-1}^{\infty} y S(t-) N(\delta t, \delta y)\right| \\
&=\left(\frac{\partial V}{\partial t}+\frac{1}{2} \sigma^{2} S^{2} \frac{\partial^{2} V}{\partial S^{2}}\right. \\
&\left.+\lambda\left[\sum_{m=1}^{M} p\left(y_{m}\right) V\left(t,\left(y_{m}+1\right) S\right)-V(t, S)\right]\right) \delta t \\
&-k \sigma S^{2}\left|\frac{\partial^{2} V}{\partial S^{2}}\right| \mathbb{E}|\delta W(t)| \\
&-k S\left|\frac{\partial^{2} V}{\partial S^{2}}\right| \mathbb{E}\left|\int_{-1}^{\infty} y S(t-) N(\delta t, \delta y)\right| .
\end{aligned}
$$

Note that $\delta W(t) \sim N(0, \delta t)$ which implies

$$
\begin{aligned}
\mathbb{E}|\delta W(t)| & =\frac{1}{\sqrt{2 \pi S t}} \int_{-\infty}^{+\infty}|x| e^{-x^{2} / 2 \delta t} d x \\
& =\frac{2}{\sqrt{2 \pi S t}} \int_{0}^{+\infty}|x| e^{-x^{2} / 2 \delta t} d x \\
& =\sqrt{\frac{2 \delta t}{\pi}} .
\end{aligned}
$$

Therefore, we have the following equation:

$$
\begin{aligned}
\frac{\partial V}{\partial t}+ & r S \frac{\partial V}{\partial S}+\frac{1}{2} \sigma^{2} S^{2} \frac{\partial^{2} V}{\partial S^{2}}-k \sigma S^{2}\left|\frac{\partial^{2} V}{\partial S^{2}}\right| \sqrt{\frac{2}{\pi \delta t}} \\
& -k S\left|\frac{\partial^{2} V}{\partial S^{2}}\right| \frac{\mathbb{E}\left|\int_{-1}^{\infty} y S(t-) N(\delta t, \delta y)\right|}{\delta t} \\
& +\lambda\left[\sum_{m=1}^{M} p\left(y_{m}\right) V\left(t,\left(y_{m}+1\right) S\right)-V(t, S)\right]-r V=0 .
\end{aligned}
$$

This completes the proof.

From Theorem 5, the following theorem is obtained.

Theorem 6. For $0 \leq t<T$, the call option price with transaction costs $\widetilde{c}(t, S(t))$ satisfies

$$
\begin{aligned}
\tilde{c}(t, S(t))=\sum_{j=0}^{\infty} e^{-\lambda(T-t)} \frac{\lambda^{j}(T-t)^{j}}{j !} \mathbb{E}[ & x N\left(\tilde{d}_{+}(\tau, x)\right) \\
& \left.-K e^{-r \tau} N\left(\tilde{d}_{-}(\tau, x)\right)\right],
\end{aligned}
$$

where

$$
\begin{aligned}
x & =S(t) \prod_{i=1}^{j}\left(U_{i}+1\right), \\
\tau & =T-t, \\
\widetilde{\sigma}^{2} & =\sigma^{2}-2 k \sigma \sqrt{\frac{2}{\pi \delta t}}-\frac{2 k \mathbb{E}\left|\int_{-1}^{\infty} y S(t-) N(\delta t, \delta y)\right|}{S \delta t}, \\
\tilde{d}_{ \pm}(\tau, x) & =\frac{\log (x / K)+\left(r-(1 / 2) \tilde{\sigma}^{2}\right) \tau}{\widetilde{\sigma} \sqrt{\tau}} .
\end{aligned}
$$

Proof. According to the definition of $\widetilde{\sigma}$ and Theorem 5, we have

$$
\begin{aligned}
\frac{\partial V}{\partial t}+ & r S \frac{\partial V}{\partial S}+\frac{1}{2} \widetilde{\sigma}^{2} S^{2} \frac{\partial^{2} V}{\partial S^{2}} \\
& +\lambda\left[\sum_{m=1}^{M} p\left(y_{m}\right) V\left(t,\left(y_{m}+1\right) S\right)-V(t, S)\right]-r V=0 .
\end{aligned}
$$

Then, it follows from Lemmas 3 and 4 that

$$
\begin{aligned}
\widetilde{c}(t, S(t))=\sum_{j=0}^{\infty} e^{-\lambda(T-t)} \frac{\lambda^{j}(T-t)^{j}}{j !} \mathbb{E}[ & x N\left(\tilde{d}_{+}(\tau, x)\right) \\
& \left.-K e^{-r \tau} N\left(\tilde{d}_{-}(\tau, x)\right)\right],
\end{aligned}
$$

which completes the proof. 
Remark 7. In [9], the standard geometric Brownian motion model has been considered and the European option pricing formula with transaction costs has been obtained through the $\Delta$-hedge. The call option price given in [9] satisfies $\widetilde{c}(t, S)=$ $S N\left(d_{1}^{\prime}\right)-K e^{-r(T-t)} N\left(d_{2}^{\prime}\right)$ where $d_{1}^{\prime}$ and $d_{2}^{\prime}$ are the same as $\widetilde{d}_{+}$ and $\widetilde{d}_{-}$defined in Theorem 6 , but $\sigma^{\prime}$ is different from $\widetilde{\sigma}$ in this paper. In terms of comparison of the call option price formula given in this paper and [9], it can be seen that the results derived in this paper extend the ones in [9] and our results are more practically useful.

\section{Conclusions}

In this paper, the European option pricing problem with transaction costs has been studied. In order to make the pricing more practical, we have chosen the Lévy jump diffusion model instead of the standard geometric Brownian motion model. By using the $\Delta$-hedged strategy, the explicit call option pricing formula has been obtained for the Lévy jump case. Our results have extended the ones in the existing literature.

\section{Conflict of Interests}

The authors declare that there is no conflict of interests regarding the publication of this paper.

\section{Acknowledgments}

This work was supported in part by the National Natural Science Foundation of China under Grant 60974030, the Shanghai Rising-Star Program of China under Grant 13QA1400100, and the Fundamental Research Funds for the Central Universities of China.

\section{References}

[1] E. Leland, "Option pricing and replication with transactions costs," The Journal of Finance, vol. 40, no. 5, pp. 1283-1301, 1985.

[2] F. Black and M. Scholes, "The pricing of options and corporate liabilities," The Journal of Political Economy, vol. 81, no. 3, pp. 637-654, 1973.

[3] J. C. Cox and S. A. Ross, "The valuation of options for alternative stochastic processes," Journal of Financial Economics, vol. 3, no. 1-2, pp. 145-166, 1976.

[4] L. O. Scott, "Pricing stock options in a jump-diffusion model with stochastic volatility and interest rates: applications of fourier inversion methods," Mathematical Finance, vol. 7, no. 4, pp. 413-426, 1997.

[5] M. Davis and A. Norman, "Portfolio selection with transaction costs," Mathematics of Operations Research, vol. 15, no. 4, pp. 676-713, 1990.

[6] R. Elliott and C. Osakwe, "Option pricing for pure jump processes with Markov switching compensators," Finance and Stochastics, vol. 10, no. 2, pp. 250-275, 2006.

[7] M. George and S. Perrakis, "Stochastic dominance bounds on derivatives prices in a multiperiod economy with proportional transaction costs," Journal of Economic Dynamics and Control, vol. 26, no. 7-8, pp. 1323-1352, 2002.
[8] P. P. Boyle, "Option replication indiscrete time with transaction costs," The Journal of Finance, vol. 47, pp. 271-293, 1992.

[9] Q. Li, Option pricing with transaction costs [M.S. thesis], Huazhong University of Science and Technology, Hubei, China, 2007.

[10] R. C. Merton, "Theory of rational option pricing," The Bell Journal of Economics and Management Science, vol. 4, no. 1, pp. 141-183, 1973.

[11] R. C. Merton, "Option pricing when underlying stock returns are discontinuous," Journal of Financial Economics, vol. 3, no. 1-2, pp. 125-144, 1976.

[12] W. Bailey and R. M. Stulz, "The pricing of stock index options in a general equilibrium model," The Journal of Financial and Quantitative Analysis, vol. 24, no. 1, pp. 1-12, 1989.

[13] G. L. Wei, L. C. Wang, and F. Han, "A gain-scheduled approach to fault-tolerant control for discrete-time stochastic delayed systems with randomly occurring actuator faults," Systems Science \& Control Engineering, vol. 1, no. 1, pp. 82-90, 2013.

[14] H. Ahmada and T. Namerikawa, "Extended Kalman filter-based mobile robot localization with intermittent measurements," Systems Science \& Control Engineering, vol. 1, no. 1, pp. 113-126, 2013.

[15] J. Hu, Z. Wang, B. Shen, and H. Gao, "Quantised recursive filtering for a class of nonlinear systems with multiplicative noises and missing measurements," International Journal of Control, vol. 86, no. 4, pp. 650-663, 2013.

[16] K. El-Tawil and A. Abou Jaoude, "Stochastic and nonlinearbased prognostic model," Systems Science \& Control Engineering, vol. 1, no. 1, pp. 66-81, 2013.

[17] M. Darouach and M. Chadli, "Admissibility and control of switched discrete-time singular systems," Systems Science \& Control Engineering, vol. 1, no. 1, pp. 43-51, 2013.

[18] P. Balasubramaniam and T. Senthilkumar, "Delay-dependent robust stabilization and $H_{\infty}$ control for uncertain stochastic T-S fuzzy systems with discrete interval and distributed timevarying delays," International Journal of Automation and Computing, vol. 10, no. 1, pp. 18-31, 2013.

[19] P. Zhou, Y. Wang, Q. Wang, J. Chen, and D. Duan, "Stability and passivity analysis for Lur'e singular systems with Markovian switching," International Journal of Automation and Computing, vol. 10, no. 1, pp. 79-84, 2013.

[20] B. Shen, Z. Wang, and Y. S. Hung, "Distributed $H_{\infty}$-consensus filtering in sensor networks with multiple missing measurements: the finite-horizon case," Automatica, vol. 46, no. 10, pp. 1682-1688, 2010.

[21] B. Shen, Z. Wang, and X. Liu, "Sampled-data synchronization control of dynamical networks with stochastic sampling," IEEE Transactions on Automatic Control, vol. 57, no. 10, pp. 26442650, 2012.

[22] B. Tang, Q. Zeng, D. He, and Y. Zhang, "Random stabilization of sampled-data control systems with nonuniform sampling," International Journal of Automation and Computing, vol. 9, no. 5, pp. 492-500, 2012.

[23] B. Shen, Z. Wang, and X. Liu, "A stochastic sampled-data approach to distributed $H_{\infty}$ Filtering in sensor networks," IEEE Transactions on Circuits and Systems I, vol. 58, no. 9, pp. 22372246, 2011.

[24] X. Liang, M. Hou, and G. Duan, "Output feedback stabilization of switched stochastic nonlinear systems under arbitrary switchings," International Journal of Automation and Computing, vol. 10, no. 6, pp. 571-577, 2013. 
[25] Z. Wang, B. Shen, and X. Liu, " $H_{\infty}$ filtering with randomly occurring sensor saturations and missing measurements," Automatica, vol. 48, no. 3, pp. 556-562, 2012.

[26] L. Stettner, "Option pricing in the CRR model with proportional transaction costs: a cone transformation approach," Applicationes Mathematicae, vol. 24, no. 4, pp. 475-514, 1997.

[27] M. Monoyios, "Option pricing with transaction costs using a Markov chain approximation," Journal of Economic Dynamics and Control, vol. 28, no. 5, pp. 889-913, 2004.

[28] P. Amster, C. G. Averbuj, and M. C. Mariani, "Stationary solutions for two nonlinear Black-Scholes type equations," Applied Numerical Mathematics, vol. 47, no. 3-4, pp. 275-280, 2003.

[29] Q. Wu, "European options with transaction costs," Journal of Tongji University, vol. 37, no. 6, 2008.

[30] K. K. Aase, "Contingent claims valuation when the security price is a combination of an Itô process and a random point process," Stochastic Processes and their Applications, vol. 28, no. 2, pp. 185-220, 1988.

[31] T. Chan, "Pricing contingent claims on stocks diven by Lévy processes," The Annals of Applied Probability, vol. 9, no. 2, pp. 504-528, 1999.

[32] H. Yan, Pricing option and EIAs when discrete dividends follow a Markov-modulated jump diffusion model [M.S. thesis], Donghua University, Shanghai, China, 2012.

[33] R. Cont and P. Tankov, Financial Modelling with Jump Processes, Chapman \& Hall, Boca Raton, Fla, USA, 2004.

[34] S. Shreve, Stochastic Analysis for Finance, Springer, Berlin, Germany, 2004. 


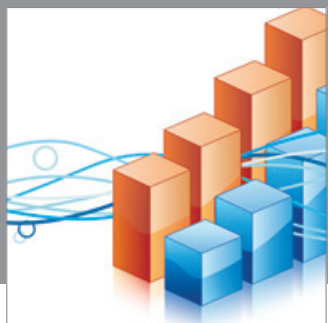

Advances in

Operations Research

mansans

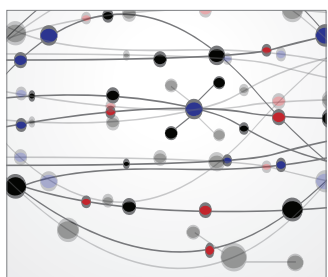

The Scientific World Journal
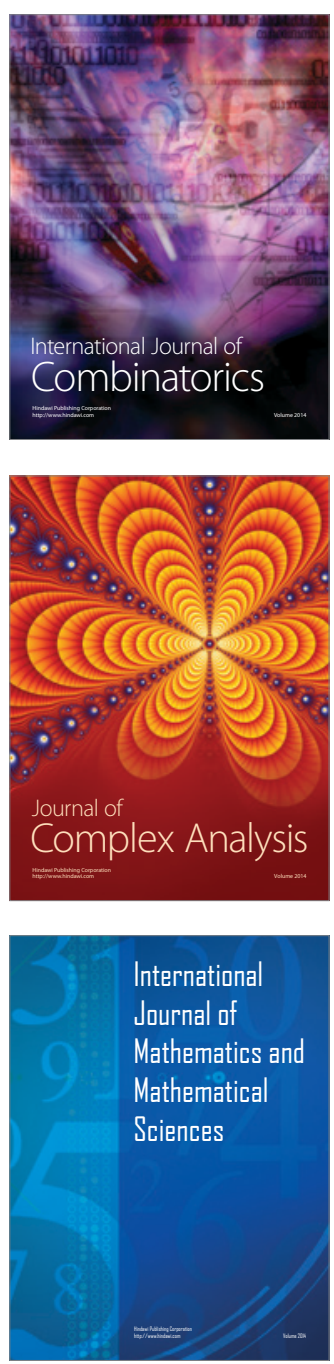
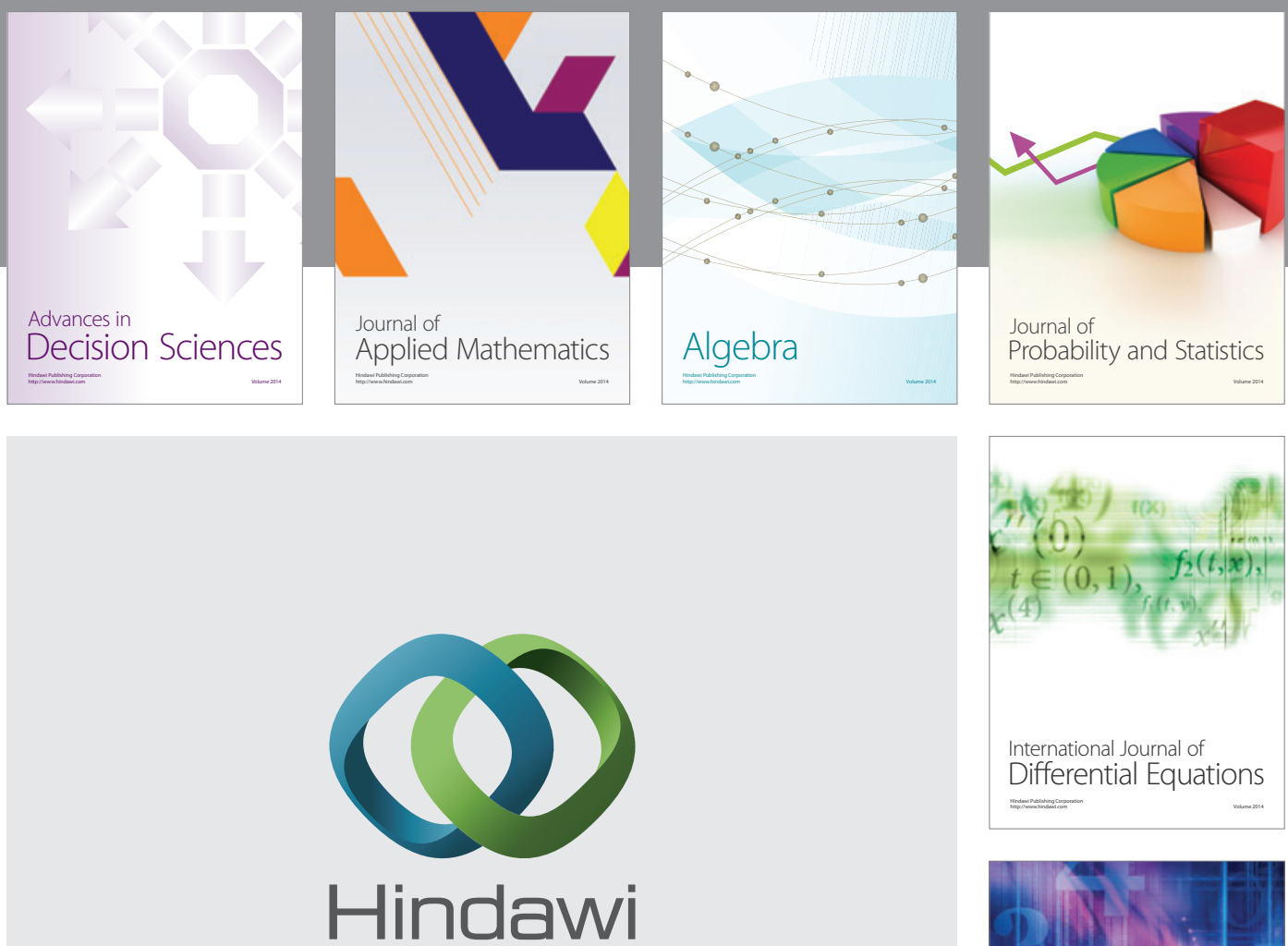

Submit your manuscripts at http://www.hindawi.com
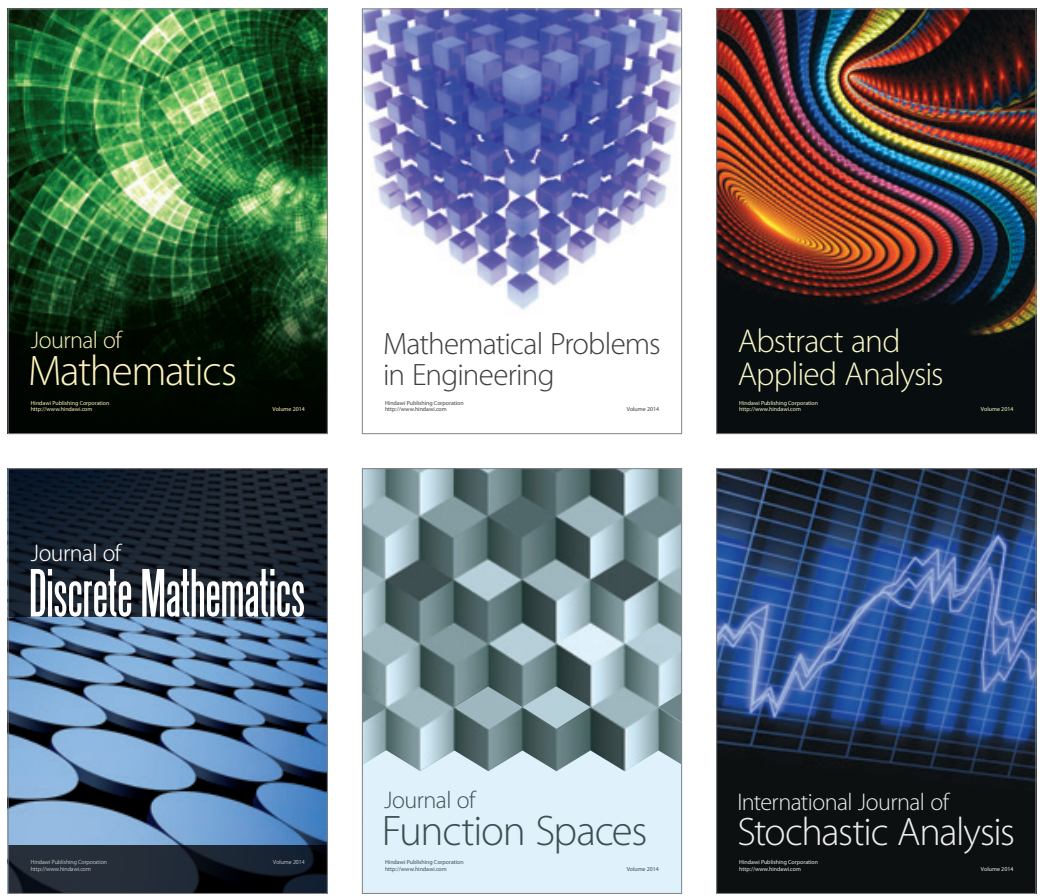

Journal of

Function Spaces

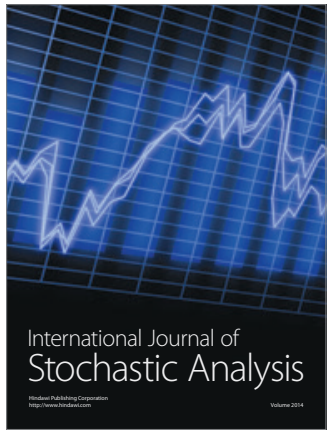

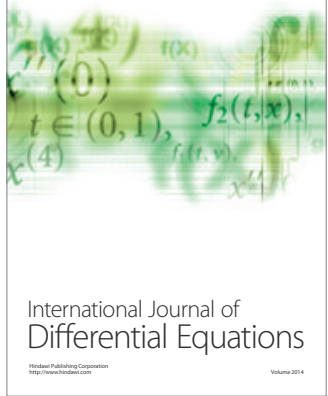
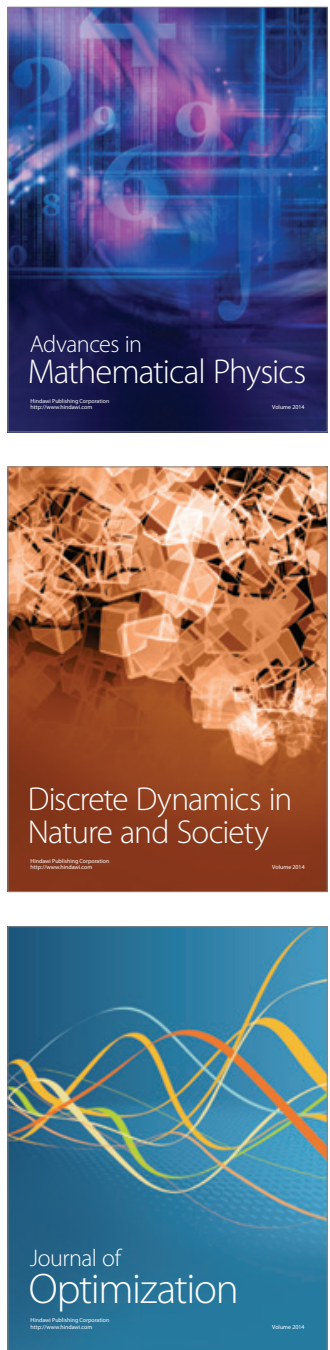